\title{
ENTIRE FUNCTIONS THAT SHARE REAL ZEROS AND REAL ONES
}

\author{
THOMAS P. CZUBIAK AND GARY G. GUNDERSEN
}

\begin{abstract}
We find all pairs of nonconstant entire functions that have the same zeros and ones (ignoring multiplicities), when the zeros and ones are all real.
\end{abstract}

We say two entire functions $f(z)$ and $g(z)$ share the value $c$ provided that $f(z)=c$ if and only if $g(z)=c$. We distinguish between sharing a value CM (counting multiplicities) and IM (ignoring multiplicities). Unless stated otherwise, all functions will be assumed to be nonconstant and entire.

It is easy to show that if $f$ and $g$ share 0 and $1 \mathrm{CM}$ and $f \neq g$, then there are entire functions $\alpha$ and $\beta$ such that

$$
f(z)=\frac{e^{2 \pi i \alpha(z)}-1}{e^{2 \pi i \beta(z)}-1} \text { and } g(z)=\frac{e^{-2 \pi i \alpha(z)}-1}{e^{-2 \pi i \beta(z)}-1} .
$$

When $f$ and $g$ have finite order, then C. F. Osgood and C. C. Yang [5, p. 410] have shown that $\beta$ is a polynomial and $\alpha$ is a polynomial in $\beta$ with rational coefficients. Thus all possible pairs are determined. For infinite order, the example $\alpha(z)=$ $z \sin \left(\pi z^{2}\right)$ and $\beta(z)=z^{2}$ shows that $\alpha$ does not even have to be a power series in $\beta$.

When $f$ and $g$ share 0 and $1 \mathrm{IM}$, the situation is more complicated. One of the authors [3, Theorem 3] has shown that $T(r, f)<(3+o(1)) T(r, g)$ and $T(r, g)<$ $(3+o(1)) T(r, f)$ as $r \rightarrow \infty$ outside a set of finite linear measure $(T(r, h)$ is the Nevanlinna characteristic function of $h$ ). M. Ozawa [6] has proven some uniqueness theorems when $f$ and $g$ have finite order, by assuming various further hypotheses on the zeros and ones. But in general, no one has come close to finding all possible pairs.

Let $\mathcal{H}$ be the class of all nonconstant entire functions which have only real zeros and real ones. We will prove the following:

THEOREM. If $f$ and $g$ are in $\mathcal{H}$ and share 0 and $1 \mathrm{IM}$, then we necessarily have one of the following six cases where $a \neq 0$ and $b$ are real constants:

1. $f=g$,

2. $f(z)=\sin ^{2}(a z+b)$ and $g(z)=-i \sin (a z+b) e^{i(a z+b)}$,

3. $f(z)=\frac{\sin ^{2}(p(a z+b))}{\sin ^{2}(a z+b)}$ and $g(z)=\frac{\sin (p(a z+b))}{\sin (a z+b)} e^{i(p-1)(a z+b)}$

$$
\text { for } p=-2 \text { and for } p=-3 \text {, }
$$

Received by the editors March 20, 1980 and, in revised form, July 7, 1980.

1980 Mathematics Subject Classification. Primary 30D35.

Key words and phrases. Entire functions, shared values. 
4. $f(z)=1-\frac{(g(z)-1)^{2}}{e^{2 p i(a z+b)}}$ and $g(z)=\frac{\sin (p(a z+b))}{\sin (a z+b)} e^{i(p-1)(a z+b)}$
for $p=3$ and for $p=4$.

REMARKS. Each case is distinct and it can be readily verified that the five unequal pairs are in $\mathcal{H}$ and share 0 and $1 \mathrm{IM}$. The following three corollaries are immediate consequences of the theorem. We note that the first part of the proof of the theorem is a direct proof of Corollary 2.

Corollary 1. If $f$ and $g$ are in $\mathcal{H}$ and share 0 and $1 \mathrm{CM}$, then $f=g$.

COROLlary 2. If $f$ and $g$ are in $\mathcal{H}$ and share 0 and $1 \mathrm{IM}$, and they are also both real on the real axis, then $f=g$.

Corollary 3. If $f(z)$ and $\sin z$ share 0 and $1 \mathrm{IM}$, then $f(z) \equiv \sin z$.

L. A. Rubel and C. C. Yang [7, p. 293] proved the statement of Corollary 3 with "IM" replaced by “CM". See also Theorem 1 of [6].

We mention the systematic study of meromorphic functions that share values by R. Nevanlinna in [4].

To prove our theorem we will apply results of Laguerre and A. Edrei.

LEMMA 1 [8, p. 266]. If $h(z)$ is entire, real for real $z$, of order less than two, and with only real zeros, then $h^{\prime}(z)$ has exactly one real zero between consecutive zeros of $h(z)$, and this zero is simple.

LEMMA 2 [2]. If $h(z)$ is entire with only real (positive) zeros and only real (positive) ones, then the order of $h$ is at most one (one-half). If further, $h(z)$ is not real for some real value of $z$, then $h$ is necessarily of one of the following two forms:

$$
h(z)=\frac{\sin (a z+b) e^{i(a z+c)}}{\sin (b-c)}, \quad \sin (b-c) \neq 0,
$$

where $a, b$, and $c$ are real constants;

$$
h(z)=\frac{\sin (p(a z+b)) e^{i(p-1)(a z+b)}}{\sin (a z+b)}
$$

where $p \neq 0,1$ is an integer and $a, b$ are real constants.

Proof of The theorem. Let $f$ and $g$ be in $\mathcal{H}$ and share 0 and 1 IM. Then by Lemma $2, \operatorname{order}(f)<1$ and $\operatorname{order}(g)<1$.

Assume first that both $f$ and $g$ are real for real $z$. Let $x_{1}$ and $x_{2}$ be consecutive zeros of $f, g$. We deduce from Lemma 1 that, in the open interval $\left(x_{1}, x_{2}\right)$, both $f$ and $g$ have exactly one of the following: (i) one double one-point, (ii) two simple one-points, or (iii) no one-points. By considering $1-f$ and $1-g$, we see that the same statement can be made with the zeros and ones interchanged. Therefore, between any two zeros (ones), all of the ones (zeros) are shared CM. We have two cases. 
In the first case, suppose that $f(x) \neq 0$ for $x>x_{3}$. It can be seen from the proof of Lemma 1 that $f^{\prime} / f$ is strictly decreasing on the interval $x>x_{3}$ (Note: In the proof of Lemma 1 it is shown that $h^{\prime} / h$ is strictly decreasing where it is defined on the real axis, for any $h$ satisfying the hypothesis of Lemma 1.). Hence, $f^{\prime}$ can have at most one zero in $\left(x_{3}, \infty\right)$. Therefore, $f$ can have at most two one-points in $\left(x_{3}, \infty\right)$. It follows from Lemma 2 that $\operatorname{order}(f)<\frac{1}{2}$ and $\operatorname{order}(g)<\frac{1}{2}$. If we further have that $f(x) \neq 0$ for $x<x_{4}$, then $f$ and $g$ must be polynomials by the Hadamard factorization theorem. This implies that $f=g$, because two polynomials that share two finite values IM are identical [1]. On the other hand, if $f$ does have infinitely many negative zeros, then by the previous paragraph, all but at most two of the one-points will be shared CM. Thus by the Hadamard factorization theorem, there exists a rational function $R(z)$ such that

$$
f-1=R(g-1) \text {. }
$$

Since $R(z)=1$ whenever $z$ is a zero of $f, g$, this implies that $R(z) \equiv 1$. Hence $f=g$.

If in the preceding paragraph we had started with the assumption that $f(x) \neq 0$ for $x<x_{4}$, instead of $f(x) \neq 0$ for $x>x_{3}$, then the same argument will yield $f=g$. Similarly, if the assumption was either $1-f(x) \neq 0$ for $x>x_{3}$, or $1-f(x) \neq 0$ for $x<x_{4}$, then the same argument with $1-f$ and $1-g$ instead of $f$ and $g$ will again yield $f=g$.

The second case is when $f$ and $g$ have infinitely many positive zeros, infinitely many negative zeros, infinitely many positive ones, and infinitely many negative ones. Our previous application of Lemma 1 to the zeros of $f, g$ and also to the zeros of $1-f, 1-g$, shows that $f$ and $g$ share 0 and $1 \mathrm{CM}$. Hence if $f \neq g$, then the result of Osgood and Yang [5, p. 410] gives

$$
f(z)=\frac{e^{n(a z+b)}-1}{e^{a z+b}-1} \text { and } g(z)=\frac{e^{-n(a z+b)}-1}{e^{-(a z+b)}-1},
$$

where $a \neq 0, b$ are constants and $n \neq 0,1$ is an integer. Now suppose $f$ and $g$ have a zero $z_{1}=(2 \pi i k / n a)-(b / a)(k$ is an integer $\neq 0, \bmod (n))$. Then $z_{2}=z_{1}+$ $(2 \pi i / a)$ is also a zero. Since $z_{1}$ and $z_{2}$ are real, we obtain $\operatorname{Re}(a)=\operatorname{Re}(b)=0$. But then $f$ and $g$ will not be real for all real $z$. Hence there are no zeros. But the same argument with $1-f$ and $1-g$ will show there are no ones. By Picard's theorem, $f$ and $g$ are constants, a case we have excluded. Therefore the assumption is false and $f=g$.

Now suppose that neither $f$ nor $g$ is real for all real $z$. Then each must have one of the forms in Lemma 2. It follows that $f=g$.

The last possibility is when, say, $f$ is real for real $z$ and $g$ is not real for some real value of $z$. Then $g$ is one of the two forms in Lemma 2 .

Case 1. $g(z)=\sin (a z+b) e^{i(a z+c)} / \sin (b-c)$ where $a \neq 0, b, c$ are real constants. Then $g$ has all simple zeros which occur when $a z+b=\pi m$ for an integer $m$ and all simple ones which occur when $a z+c=\pi N$ for an integer $N$. Since the distance between any two consecutive zeros and any two consecutive ones is equal, there is exactly one zero between every two ones. By applying Lemma 1 to $1-f$, 
we see that $f$ has all double zeros. This means

$$
f(z)=(g(z))^{2} e^{A z+B} \quad \text { for constants } A, B .
$$

Since $f$ is real on the real axis, this means that there are real constants $C, D$, and an integer $k$ so that

$$
f(z)=\frac{\sin ^{2}(a z+b)}{\sin ^{2}(b-c)} e^{C z+D+\pi i k} .
$$

Now $g(z)=1 \Rightarrow \sin ^{2}(a z+b)=\sin ^{2}(b-c) \Rightarrow e^{C z+D+\pi i k}=1$. Since the ones are all real, it follows that $e^{C z+D+\pi i k} \equiv 1$. Further, since the ones of $f$ can only occur when $a z+c=\pi N$, this means that $\sin ^{2}(b-c)=1$. Thus

$$
f(z)=\sin ^{2}(a z+b) \text { and } g(z)=-i \sin (a z+b) e^{i(a z+b)} \text {. }
$$

Case 2. $g(z)=\sin (p(a z+b)) e^{i(p-1)(a z+b)} / \sin (a z+b)$ where $p \neq 0,1$ is an integer and $a \neq 0, b$ are real constants. Then $g$ has all simple zeros which occur when $p(a z+b)=\pi N$ for an integer $N \neq 0(\bmod p)$, and all simple ones which occur when $(p-1)(a z+b)=\pi m$ for an integer $m \neq 0(\bmod (p-1))$.

Suppose first $p<0$. There is at most one zero between consecutive ones. Applying Lemma 1 to $1-f$ shows that $f$ can have only double zeros. Then for constants $c, d$

$$
f(z)=(g(z))^{2} e^{c z+d} .
$$

Since $f$ is real on the real axis, this means there are real constants $A, B$ and an integer $k$ so that:

$$
f(z)=\frac{\sin ^{2}(p(a z+b)) e^{A z+B+k \pi i}}{\sin ^{2}(a z+b)} .
$$

Since the ones are real, this further reduces to

$$
f(z)=\frac{\sin ^{2}(p(a z+b))}{\sin ^{2}(a z+b)} .
$$

If $p=-1$ then $f=1$, the excluded constant case. The cases $p=-2$ and $p=-3$ give item 3 in the theorem. Now assume $p<-4$. If $a z+b=\pi /(p+1)$, then $f(z)=1$. Hence $g(z)=1$, and from the preceding paragraph, this means that $(p-1) /(p+1)=m$ for some integer $m$. This cannot happen for $p<-4$.

Now suppose $p>2$. There is at most one one-point between zeros. Hence by Lemma $1, f$ can have only double ones. Then for constants $c$ and $d$ :

$$
f(z)-1=-(g(z)-1)^{2} e^{c z+d} \text {. }
$$

By considering two different zeros of $f, g$ we obtain $\operatorname{Re}(c)=\operatorname{Re}(d)=0$. Then for real constants $A, B$,

$$
f(z)=1-(g(z)-1)^{2} e^{i(A z+B)} .
$$

From this we obtain

$$
f(z)=1-(w(z))^{2} \exp [i(A z+B+2 p(a z+b))]
$$


where $w(z)=\sum_{k=1}^{p-1} \exp [i(p-2 k)(a z+b)]$ is real for real $z$. Hence

$$
\exp (i(A z+B)) \equiv \exp (-2 p i(a z+b)) \text {. }
$$

Substituting this into (3),

$$
f(z)=1-(g(z)-1)^{2} e^{-2 p i(a z+b)} .
$$

Then for $p=2, g(z)=e^{2 i(a z+b)}+1$ and $f(z) \equiv 0$. The cases $p=3$ and $p=4$ give item 4 in the theorem. Now assume $p>5$. $f(z)=0 \Leftrightarrow(g(z)-1)^{2}=e^{2 p i(a z+b)}$. Thus $f(z)=g(z)=0$ whenever $a z+b=\pi /(p-2)$. Therefore, $p=N(p-2)$ for some integer $N$. This is impossible for $p>5$.

The proof of the theorem is now complete.

\section{REFERENCES}

1. W. Adams and E. Straus, Non-Archimedian analytic functions taking the same values at the same points, Illinois J. Math. 15 (1971), 418-424.

2. A. Edrei, Meromorphic functions with three radially distributed values, Trans. Amer. Math. Soc. 78 (1955), 276-293.

3. G. G. Gundersen, Meromorphic functions that share three or four values, J. London Math. Soc. 20 (1979), 457-466.

4. R. Nevanlinna, Le théorème de Picard-Borel et la théorie des fonctions méromorphes, Gauthier-Villars, Paris, 1929.

5. C. F. Osgood and C. C. Yang, On the quotient of two integral functions, J. Math. Anal. Appl. 54 (1976), 408-418.

6. M. Ozawa, Unicity theorems for entire functions, J. Analyse Math. 30 (1976), 411-420.

7. L. A. Rubel and C. C. Yang, Interpolation and unavoidable families of meromorphic functions, Michigan Math. J. 20 (1973), 289-296.

8. E. C. Titchmarsh, The theory of functions, 2nd ed., Clarendon Press, Oxford, 1968.

Department of Mathematics, University of New Orleans, New Orleans, Louisiana 70122 (Current address of G. G. Gundersen)

Current address (T. P. Czubiak): 4916 S. Prieur, New Orleans, Louisiana 70125 